\title{
A General Form for Calculating Residual Stresses Detected by Using the Holographic Blind-hole Method
}

\author{
by S. T. Lin, C. T. Hsieh and C. K. Lee
}

\begin{abstract}
A general form used for analyzing residual stresses measured using the holographic blind-hole method is introduced in this paper. Adopting the general form presented, the residual stresses can be obtained using three relative displacements measured from a single interference fringe pattern. Even for the case in which phase-shifting holographic interferometry is not employed, interpolating calculations for determining the fringe orders are not needed, since the choice of data points becomes more flexible when using this general form. Two experiments, the first one carried out by the authors and the second one published previously, are used to illustrate the applicability and usefulness of this general form. Suggestions for the applications of this general form are also established via the upper bound error estimations.
\end{abstract}

\section{KEY WORDS—Holography, residual stresses}

\section{Introduction}

Holographic interferometry was first used by Antonov ${ }^{1}$ to measure the out-of-plane displacement field due to blindhole drilling (with the sensitivity vector of the interference fringe pattern set normal to the detected surface). An outof-plane displacement of a point around the blind hole was used to determine uniaxial residual stresses. Bass, Schmitt and Ahrens ${ }^{2}$ then adopted this method to determine in-plane residual stresses on the surface of a solid body. A displacement vector solved using three interference fringe patterns was required to determine the stresses. At about the same time, Nelson and McCrickerd ${ }^{3}$ canceled the out-of-plane and tangential displacements by taking the difference of fringe orders of two diametrically opposite points lying on the projection of the sensitivity vector. Three radial displacements extracted from three interference fringe patterns were required to determine the residual stresses. To simplify the optical setup and facilitate application of the holographic blind-hole method, a single-axis holographic blind-hole method was then proposed by Makino and Nelson ${ }^{4}$ and Nelson et al. ${ }^{5}$ In their single-axis holographic blind-hole method, the effect of the out-of-plane displacement field was canceled by subtracting the fringe order of one point from its diametrically opposite point. Three in-plane displacements obtained

S. T. Lin (SEM Member) is Associate Professor, Department of Mechanical Engineering, National Taipel University of Technology, Republic of China. C. T. Hsieh is Lecturer, Department of Mechanical Engineering, St. Jone's and St. Mary's Institute of Technology, Republic of China. C. K. Lee is Professor, Institute of Applied Mechanics, National Taiwan University, Republic of China.

Original manuscript submitted: February 7, 1997.

Final manuscript received: June 29, 1998. from one interference fringe pattern were sufficient to recover the residual stresses. Two other single-axis holographic blind-hole methods were later proposed by Lin, Hsieh and $\mathrm{Hu}^{6}$ to further simplify the methodology. The first one canceled the in-plane displacement field by adding the fringe orders of two diametrically opposite points. Three relative out-of-plane displacements deduced from a single interference fringe pattern were sufficient to determine the residual stresses. The second one was similar to that proposed by Antonov ${ }^{1}$ but used two relative out-of-plane displacements to determine the residual stresses. Theoretical results obtained by the researchers mentioned above ${ }^{4-6}$ compared well with their respective experimental data. However, interpolation was employed to extract the fringe orders of some data points in order to perform accurate measurement.

Although the phase-shifting holographic blind-hole method $^{7}$ is a convenient and precise technique for determining residual stresses, the expensive equipment and software required pose a high threshold to justify widespread use. To further spread the adoption of the holographic blindhole method, it is the intention of this paper to create a more general form to facilitate the analysis of the residual stresses measured using the holographic blind-hole method. With this more general form, three relative displacements obtained from a single interference fringe pattern are sufficient to determine the residual stresses released from the drilling of a blind hole. Interpolation is not needed to determine the fringe orders because the choice of data points becomes more flexible in this new method. It is expected that with the introduction of this more general form, the holographic blind-hole method will become easier to implement whether a phase-shifting technique is employed or not.

\section{Displacement Field from Blind-hole Drilling}

Since residual stresses on the surface of a body can be separated into a mean stress $\sigma_{m}$ and a pure shear stress $\sigma_{s}$, the principal stresses can be expressed as

$$
\sigma_{\max }=\sigma_{m}+\sigma_{s}
$$

and

$$
\sigma_{\min }=\sigma_{m}-\sigma_{s},
$$

where $\sigma_{\max }$ and $\sigma_{\min }$ are maximum and minimum principal stresses, respectively. Let

$$
\sigma_{m}=C_{m} \cdot E
$$

and

$$
\sigma_{s}=C_{s} \cdot E,
$$




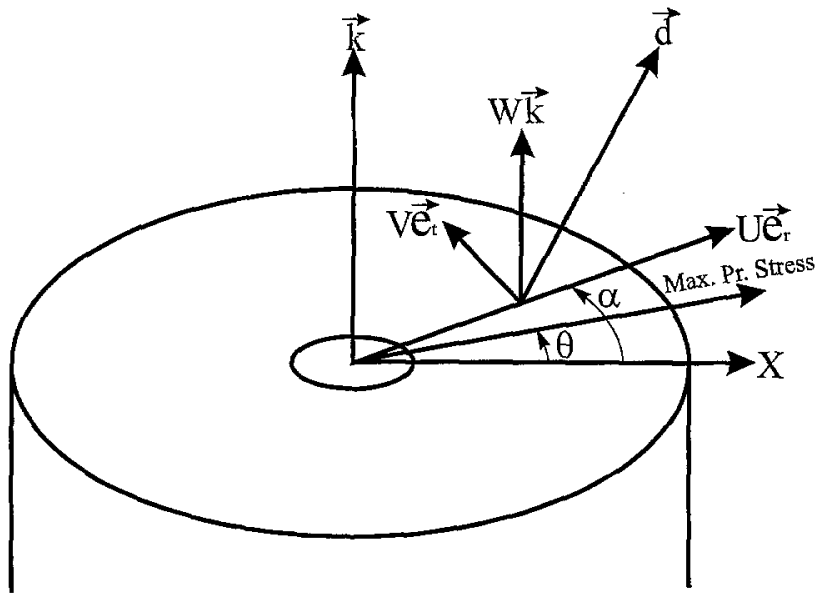

Fig. $1-$ Coordinates and displacement vector in an axisymmetric body

where $E$ is the Young's modulus of an isotropic material and $C_{m}$ and $C_{s}$ are two dimensionless coefficients. Once a blind hole is drilled into the surface of the solid body, the mean stress and shear stress will be relieved simultaneously. Referring to Fig. 1 and $\mathrm{Lin}, \mathrm{Hsieh}$ and $\mathrm{Hu},{ }^{6}$ the displacement field can be expressed as

$$
\begin{aligned}
\vec{d} & =\left[C_{m} U_{m}+C_{s} U_{s} \cos 2(\alpha-\theta)\right] \vec{e}_{r} \\
& -C_{s} V_{s} \sin 2(\alpha-\theta) \vec{e}_{t} \\
& +\left[C_{m} W_{m}+C_{s} W_{s} \cos 2(\alpha-\theta)\right] \vec{k}
\end{aligned}
$$

where $\vec{e}_{r}, \vec{e}_{t}$, and $\vec{k}$ are the unit vectors of the radial, tangential and normal directions, respectively; $\theta$ is the angle measured from the $x$-axis to the direction of the maximum principal stress; and $U_{m}, U_{s}, V_{s}, W_{m}$ and $W_{s}$ are displacement constants calculated by finite element analysis ${ }^{6}$ and tabulated in Tables 1-5. To get the maximum benefit from these tables, nondimensional variables $H=h / D_{0}, D=d / D_{0}$ and Poisson's ratio $v$ are adopted in these tables, and $h, D_{0}$ and $d$ are used to represent hole depth, hole diameter and general diameter, respectively. It should be noted that the displacement constants used in Tables 1-5 are also nondimensionalized with respect to the hole diameter $D_{0}$.

\section{More General Form}

The physical meaning of the interference fringe pattern obtained from the holographic interferometry can be expressed as

$$
\vec{K} \cdot\left(\vec{d}\left(p_{i 1}\right)-\vec{d}\left(p_{i 2}\right)\right)=\Delta N_{i}\left(p_{i 1}, p_{i 2}\right) \lambda,
$$

where $\lambda$ is the laser wave length and, referring to Fig. 2, $\vec{K}$ is the sensitivity vector, and $\vec{d}\left(p_{i 1)}\right.$ and $\vec{d}\left(p_{i 2}\right)$ are the displacement vectors of points $p_{i 1}$ and $p_{i 2}$, respectively. In addition, $\Delta N_{i}\left(p_{i 1}, p_{i 2}\right)=N\left(p_{i 1}\right)-N\left(p_{i 2}\right)$ is the difference of fringe orders of points $p_{i 1}$ and $p_{i 2}$. Substituting eq (3) into eq (4) with $i=1,2,3$, we have the following compact equation:

$$
\left[a_{i j}\left(p_{i 1}, p_{i 2}, \alpha_{i}\right)\right]\left[c_{j}\right]=\left[\Delta N_{i}\left(p_{i 1}, p_{i 2}\right)\right] \lambda
$$

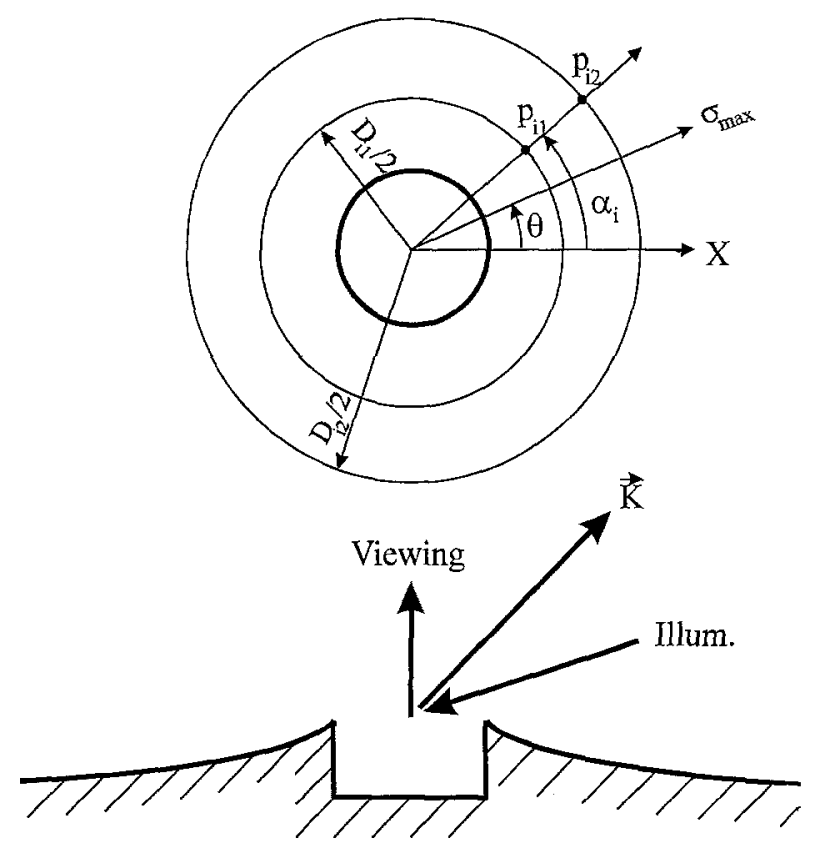

Fig. 2-Sensitivity vector and the points around a blind hole

where $i, j$ are from 1 to 3 and

$$
\begin{aligned}
a_{i 1} & =\left(\Delta U_{m i} \vec{e}_{r}\left(\alpha_{i}\right)+\Delta W_{m i} \vec{k}\right) \cdot \vec{K} \\
a_{i 2} & =\left(\Delta U_{s i} \cos 2 \alpha_{i} \vec{e}_{r}\left(\alpha_{i}\right)-\Delta V_{s i} \sin 2 \alpha_{i} \vec{e}_{t}\left(\alpha_{i}\right)\right. \\
& \left.+\Delta W_{s i} \cos 2 \alpha_{i} \vec{k}\right) \cdot \vec{K} \\
a_{i 3} & =\left(\Delta U_{s i} \sin 2 \alpha_{i} \vec{e}_{r}\left(\alpha_{i}\right)+\Delta V_{s i} \cos 2 \alpha_{i} \vec{e}_{t}\left(\alpha_{i}\right)\right. \\
& \left.+\Delta W_{s i} \sin 2 \alpha_{i} \vec{k}\right) \cdot \vec{K} .
\end{aligned}
$$

Also, we have

$$
\begin{aligned}
& c_{1}=C_{m} \\
& c_{2}=C_{s} \cos 2 \theta \\
& c_{3}=C_{s} \sin 2 \theta .
\end{aligned}
$$

The relative displacement constants are

$$
\begin{aligned}
\Delta U_{m i} & =U_{m}\left(p_{i 1}\right)-U_{m}\left(p_{i 2}\right) \\
\Delta U_{s i} & =U_{s}\left(p_{i 1}\right)-U_{s}\left(p_{i 2}\right) \\
\Delta V_{s i} & =V_{s}\left(p_{i 1}\right)-V_{s}\left(p_{i 2}\right) \\
\Delta W_{m i} & =W_{m}\left(p_{i 1}\right)-W_{m}\left(p_{i 2}\right) \\
\Delta W_{s i} & =W_{s}\left(p_{i 1}\right)-W_{s}\left(p_{i 2}\right) .
\end{aligned}
$$

Referring to Fig. 2, it should be noted that $p_{i 1}$ and $p_{i 2}$ lie on the same radial line with an angle $\alpha_{i}$ measured from the $x$-axis, and $p_{i 1}$ and $p_{i 2}$ lie on the circles with diameters $D_{i 1}$ and $D_{i 2}$, respectively. Furthermore, the diameters of $D_{i 1}$, $i=1,2,3$, need not be the same as each other, and this is also the condition of $D_{i 2}, i=1,2,3$.

\section{Example 1}

A specimen made of aluminum alloy was used to produce residual stresses. Referring to Fig. 3, the specimen was first loaded and then fixed. Strain gage 1 was used to monitor the tensile stress at point A due to the fixing force. Using 
TABLE 1-DISPLACEMENT CONSTANT $U_{m} / D_{0}$

\begin{tabular}{cccccccccccccc}
\hline$H$ & \multicolumn{3}{c}{0.6} & \multicolumn{3}{c}{0.8} & \multicolumn{3}{c}{$\mathbf{1 . 0}$} & \multicolumn{3}{c}{1.2} \\
$D \backslash \nu$ & .25 & .30 & .35 & .25 & .30 & .35 & .25 & .30 & .35 & .25 & .30 \\
\hline 1.8 & .2955 & .3027 & .3088 & .3321 & .3420 & .3508 & .3514 & .3631 & .3738 & .3619 & .3746 & .3865 \\
2.2 & .2204 & .2256 & .2299 & .2591 & .2666 & .2731 & .2819 & .2909 & .2991 & .2952 & .3054 & .3148 \\
2.6 & .1686 & .1724 & .1755 & .2059 & .2116 & .2166 & .2303 & .2375 & .2440 & .2460 & .2542 & .2618 \\
3.0 & .1326 & .1355 & .1379 & .1667 & .1712 & .1751 & .1911 & .1969 & .2020 & .2081 & .2148 & .2210 \\
3.4 & .1072 & .1095 & .1113 & .1376 & .1412 & .1443 & .1609 & .1656 & .1698 & .1782 & .1838 & .1889
\end{tabular}

NOTE: $H=h / D_{0}$ and $D=d / D_{0}$, where $D_{0}$ is hole diameter, $h$ is hole depth, and $d$ is general diameter. Since the hole depths of $H<0.6$ are seldom used, the displacement constants corresponding to these values are not listed here.

TABLE 2-DISPLACEMENT CONSTANT $U_{s} / D_{0}$

\begin{tabular}{ccccccccccccc}
\hline$H$ & \multicolumn{3}{c}{0.6} & \multicolumn{3}{c}{0.8} & \multicolumn{3}{c}{$\mathbf{1 . 0}$} & \multicolumn{3}{c}{1.2} \\
\cline { 2 - 7 } & .25 & .30 & .35 & .25 & .30 & .35 & .25 & .30 & .35 & .25 & .30 \\
\hline 1.8 & .5480 & .5564 & .5641 & .6615 & .6682 & .6741 & .7408 & .7453 & .7489 & .7980 & .8003 & .8014 \\
2.2 & .4045 & .4123 & .4195 & .5092 & .5157 & .5214 & .5861 & .5908 & .5944 & .6432 & .6460 & .6473 \\
2.6 & .3053 & .3121 & .3185 & .3974 & .4035 & .4089 & .4694 & .4741 & .4777 & .5249 & .5279 & .5296 \\
3.0 & .2371 & .2430 & .2486 & .3163 & .3218 & .3267 & .3816 & .3861 & .3897 & .4342 & .4373 & .4392 \\
3.4 & .1895 & .1945 & .1993 & .2571 & .2620 & .2664 & .3155 & .3197 & .3231 & .3642 & .3673 & .3694
\end{tabular}

NOTE: $H=h / D_{0}$ and $D=d / D_{0}$, where $D_{0}$ is hole diameter, $h$ is hole depth, and $d$ is general diameter. Since the hole depths of $H<0.6$ are seldom used, the displacement constants corresponding to these values are not listed here.

TABLE 3-DISPLACEMENT CONSTANT $V_{s} / D_{0}$

\begin{tabular}{|c|c|c|c|c|c|c|c|c|c|c|c|c|}
\hline$H$ & \multicolumn{3}{|c|}{0.6} & \multicolumn{3}{|c|}{0.8} & \multicolumn{3}{|c|}{1.0} & \multicolumn{3}{|c|}{1.2} \\
\hline$D \backslash \cup$ & .25 & .30 & .35 & .25 & .30 & .35 & .25 & .30 & .35 & .25 & .30 & .35 \\
\hline 1.8 & .2017 & .1800 & .1578 & .2459 & .2185 & .1906 & .2788 & .2468 & .2144 & .3038 & .2682 & .2321 \\
\hline 2.2 & .1342 & .1169 & .0990 & .1679 & .1459 & .1234 & .1944 & .1685 & .1422 & .2153 & .1862 & .1569 \\
\hline 2.6 & .0987 & .0848 & .0704 & .1256 & .1078 & .0894 & .1477 & .1265 & .1049 & .1657 & .1418 & .1175 \\
\hline 3.0 & .0775 & .0663 & .0546 & .1001 & .0854 & .0703 & .1191 & .1015 & .0836 & .1350 & .1150 & .0947 \\
\hline 3.4 & .0640 & .0548 & .0452 & .0835 & .0713 & .0588 & .1003 & .0856 & .0706 & .1148 & .0979 & .0808 \\
\hline
\end{tabular}

NOTE: $H=h / D_{0}$ and $D=d / D_{0}$, where $D_{0}$ is hole diameter, $h$ is hole depth, and $d$ is general diameter. Since the hole depths of $H<0.6$ are seldom used, the displacement constants corresponding to these values are not listed here.

TABLE 4-DISPLACEMENT CONSTANT $W_{m} / D_{0}$

\begin{tabular}{|c|c|c|c|c|c|c|c|c|c|c|c|c|}
\hline$H$ & & 0.6 & & & 0.8 & & & 1.0 & & & 1.2 & \\
\hline$D \backslash \mathcal{v}$ & .25 & .30 & .35 & .25 & .30 & .35 & .25 & .30 & .35 & .25 & .30 & .35 \\
\hline $\begin{array}{l}1.8 \\
2.2 \\
2.6 \\
3.0 \\
3.4\end{array}$ & $\begin{array}{l}.0589 \\
.0246 \\
.0040 \\
-.0084 \\
-.0164\end{array}$ & $\begin{array}{l}.0601 \\
.0250 \\
.0039 \\
-.0088 \\
-.0169\end{array}$ & $\begin{array}{c}.0624 \\
.0267 \\
.0052 \\
-.0078 \\
-.0161\end{array}$ & $\begin{array}{l}.0688 \\
.0351 \\
.0111 \\
-.0053 \\
-.0166\end{array}$ & $\begin{array}{l}.0712 \\
.0363 \\
.0117 \\
-.0052 \\
-.0168\end{array}$ & $\begin{array}{l}.0743 \\
.0385 \\
.0133 \\
-.0040 \\
-.0160\end{array}$ & $\begin{array}{l}.0691 \\
.0393 \\
.0156 \\
-.0024 \\
-.0158\end{array}$ & $\begin{array}{l}.0711 \\
.0401 \\
.0155 \\
-.0031 \\
-.0169\end{array}$ & $\begin{array}{l}.0760 \\
.0440 \\
.0187 \\
-.0005 \\
-.0148\end{array}$ & $\begin{array}{l}.0627 \\
.0376 \\
.0158 \\
-.0022 \\
-.0165\end{array}$ & $\begin{array}{l}.0647 \\
.0385 \\
.0158 \\
-.0028 \\
-.0177\end{array}$ & $\begin{array}{l}.0707 \\
.0435 \\
.0200 \\
.0007 \\
-.0146\end{array}$ \\
\hline
\end{tabular}

NOTE: $H=h / D_{0}$ and $D=d / D_{0}$, where $D_{0}$ is hole diameter, $h$ is hole depth, and $d$ is general diameter. Since the hole depths of $H<0.6$ are seldom used, the displacement constants corresponding to these values are not listed here.

TABLE 5-DISPLACEMENT CONSTANT $W_{S} / D_{0}$

\begin{tabular}{|c|c|c|c|c|c|c|c|c|c|c|c|c|}
\hline \multirow{2}{*}{$\begin{array}{c}H \\
D \backslash \nu\end{array}$} & \multicolumn{3}{|c|}{0.6} & \multicolumn{3}{|c|}{0.8} & \multicolumn{3}{|c|}{1.0} & \multicolumn{3}{|c|}{1.2} \\
\hline & .25 & .30 & .35 & .25 & .30 & .35 & .25 & .30 & .35 & .25 & .30 & .35 \\
\hline $\begin{array}{l}1.8 \\
2.2 \\
2.6 \\
3.0 \\
3.4\end{array}$ & $\begin{array}{l}.0477 \\
.0073 \\
-.0112 \\
-.0191 \\
-.0220\end{array}$ & $\begin{array}{l}.0753 \\
.0284 \\
.0052 \\
-.0060 \\
-.0114\end{array}$ & $\begin{array}{l}.1029 \\
.0499 \\
.0220 \\
.0074 \\
-.0006\end{array}$ & $\begin{array}{l}.0895 \\
.0393 \\
.0103 \\
-.0058 \\
-.0147\end{array}$ & $\begin{array}{l}.1227 \\
.0652 \\
.0310 \\
.0110 \\
-.0009\end{array}$ & $\begin{array}{l}.1555 \\
.0912 \\
.0518 \\
.0279 \\
.0131\end{array}$ & $\begin{array}{l}.1224 \\
.0685 \\
.0334 \\
.0111 \\
-.0030\end{array}$ & $\begin{array}{l}.1594 \\
.0979 \\
.0572 \\
.0307 \\
.0133\end{array}$ & $\begin{array}{l}.1958 \\
.1268 \\
.0808 \\
.0502 \\
.0297\end{array}$ & $\begin{array}{l}.1477 \\
.0929 \\
.0547 \\
.0283 \\
.0102\end{array}$ & $\begin{array}{l}.1873 \\
.1246 \\
.0807 \\
.0500 \\
.0285\end{array}$ & $\begin{array}{l}.2259 \\
.1557 \\
.1063 \\
.0714 \\
.0466\end{array}$ \\
\hline
\end{tabular}

NOTE: $H=h / D_{0}$ and $D=d / D_{0}$, where $D_{0}$ is hole diameter, $h$ is hole depth, and $d$ is general diameter. Since the hole depths of $H<0.6$ are seldom used, the displacement constants corresponding to these values are not listed here. 


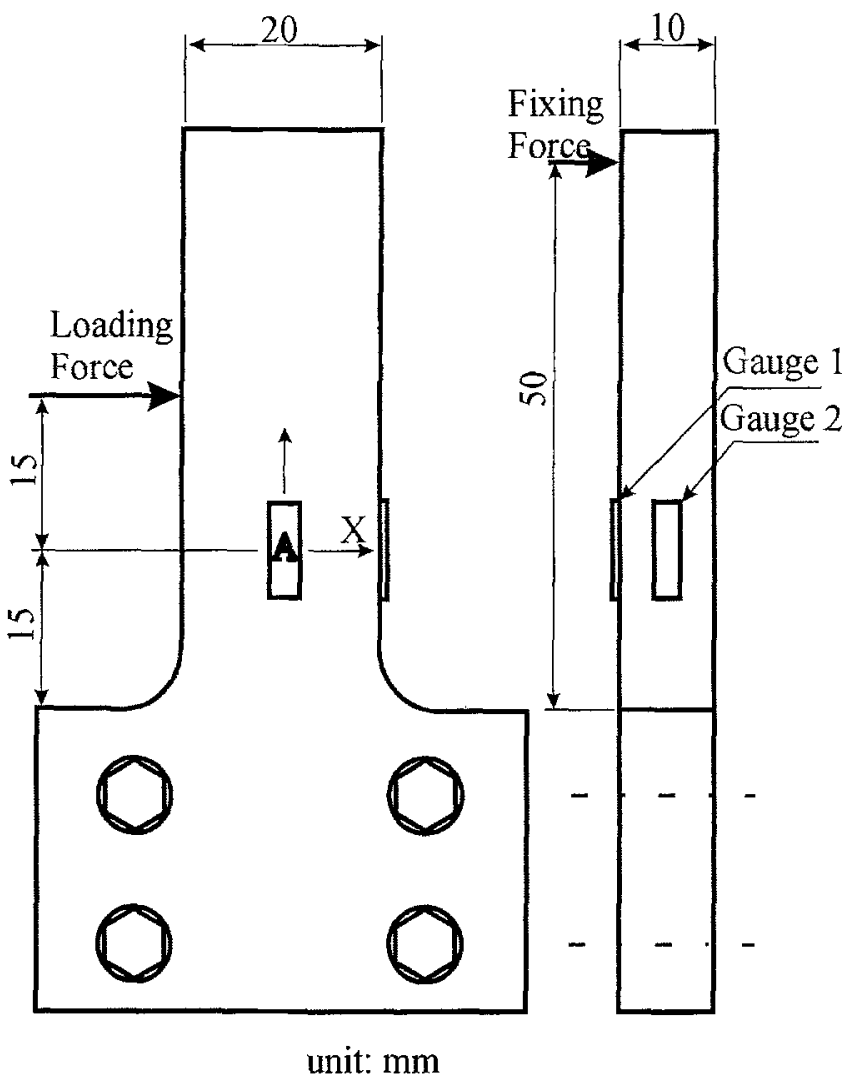

Fig. 3-Specimen
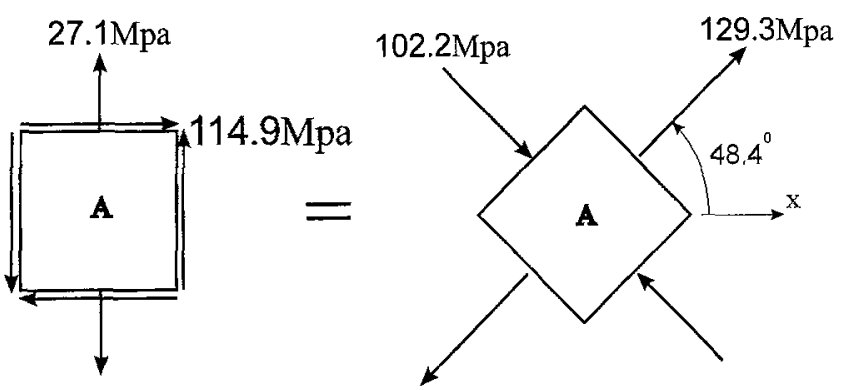

Fig. 4-Residual stresses at point $A$

beam theory, ${ }^{8}$ strain gage 2 was used to measure the loading force that was then used to calculate the pure shear stress at point $\mathrm{A}$ due to this force. These two stresses at point $\mathrm{A}$, a pure shear stress and a tensile stress, are shown in Fig. 4. A Du Pont phótopolymer hologram was used to record the initial image of the specimen, which was then drilled using a RS-200 milling guide produced by Measurement Group. The resulting interference fringe pattern was immediately viewed through the hologram in real time and is shown in Figs. 5 and 6 . The test parameters and elastic modulus include a wavelength $\lambda$ of $0.488 \mu \mathrm{m}$, a sensitivity vector $\vec{K}$ of $0.707 \vec{i}$ $+1.707 \vec{k}$, a hole diameter $D_{0}$ of $1.85 \mathrm{~mm}$, a hole depth $H$ of 0.76 , a Poisson's ratio $v$ of 0.33 and an elastic modulus $E$ of $72 \mathrm{Gpa}$.

Figure 5, which is first used to illustrate the use of eq (5), also shows the hole boundary; the $x$-axis; the angles $\alpha_{1}=7$ deg, $\alpha_{2}=60 \mathrm{deg}$, and $\alpha_{3}=-45 \mathrm{deg}$ (noting that these angles are selected arbitrarily); the selected data points $P_{i 1}$ and $p_{i 2} ;$ and the fringe order $\left[\Delta N_{i}\left(p_{i 1}, p_{i 2}\right)\right]=[0,2,-2]^{T}$. The hole dimensions and Poisson's ratio shown above, and $D_{i 1}$ and $D_{i 2}$ measured directly from Fig. 5, were put into

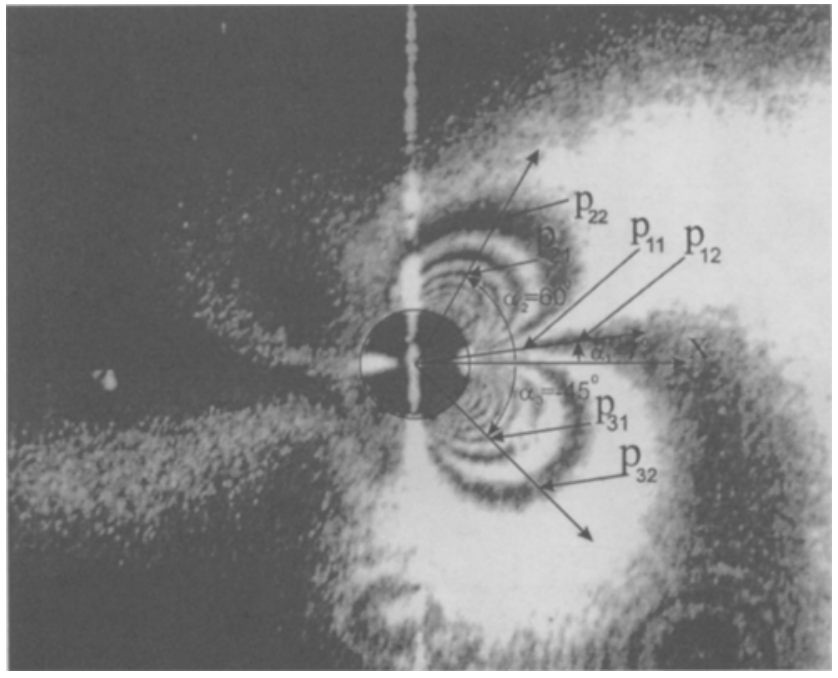

Fig. 5-Interference fringe pattern obtained from blind-hole drilling

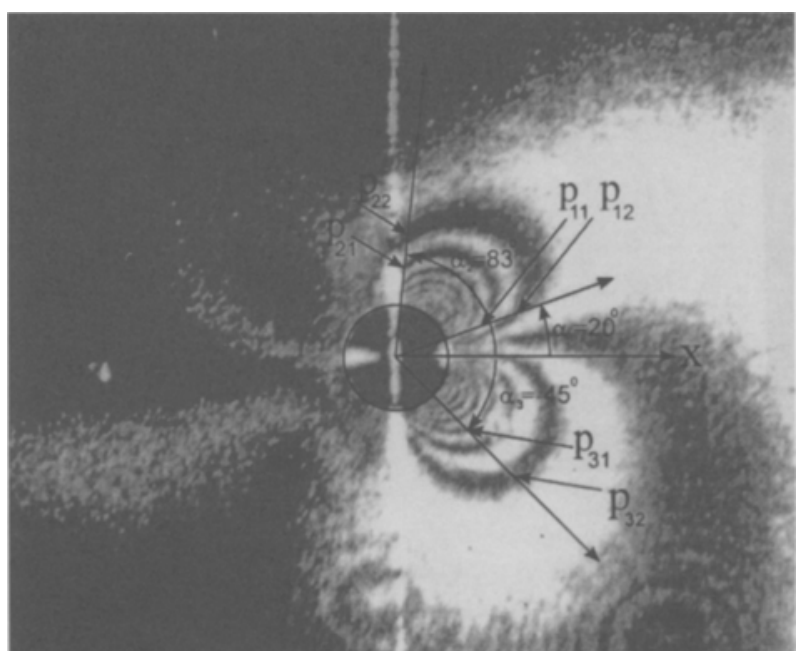

Fig. 6-Interference fringe pattern obtained from blind-hole drilling

\section{TABLE 6}

\begin{tabular}{cccc}
\hline & $i=1$ & $i=2$ & $i=3$ \\
\hline$\Delta U_{m i}$ & .2475 & .2853 & .2960 \\
$\Delta U_{s i}$ & .4891 & .5637 & .5840 \\
$\Delta V_{s i}$ & .1641 & .1930 & .1943 \\
$\Delta W_{m i}$ & .1073 & .1241 & .1273 \\
$\Delta W_{s i}$ & .1545 & .1798 & .1820
\end{tabular}

Tables 1-5 to get the relative displacement constants. These constants are shown in Table 6.

The dimensions of the above constants are in millimeters ( $\mathrm{mm})$. Substituting the above data into eqs (5), (2) and (1), we have $\sigma_{\max }=143 \mathrm{Mpa}, \sigma_{\min }=-110 \mathrm{Mpa}, \theta=54 \mathrm{deg}$.

Figure 6, which is also used to illustrate the use of eq (5), shows the same fringe pattern with the different angles $\alpha_{1}=20 \mathrm{deg}, \alpha_{2}=83 \mathrm{deg}$, and $\alpha_{3}=-45 \mathrm{deg}$, data points $P_{i 1}$ and $p_{i 2}$, and fringe order $\left[\Delta N_{i}\left(p_{i 1}, p_{i 2}\right)\right]=[1,1,-2]^{T}$. Putting the hole dimensions and Poisson's ratio shown above, together with $D_{i 1}$ and $D_{i 2}$ measured directly from Fig. 6, into Tables 1-5, we have the relative displacement constants, with units in millimeters, in Table 7. 


\begin{tabular}{cccc}
\hline & $i=1$ & $i=2$ & $i=3$ \\
\hline$\Delta U_{m i}$ & .2499 & .2283 & .2960 \\
$\Delta U_{s i}$ & .4703 & .4325 & .5840 \\
$\Delta V_{s i}$ & .2126 & .2090 & .1943 \\
$\Delta W_{m i}$ & .1077 & .0993 & .1273 \\
$\Delta W_{s i}$ & .1780 & .1698 & .1820
\end{tabular}

Substituting the above data into eqs (5), (2) and (1), we have $\sigma_{\max }=133 \mathrm{Mpa}, \sigma_{\min }=-111 \mathrm{Mpa}, \theta=50 \mathrm{deg}$.

\section{Example 2}

To further verify the method presented in this paper, experiment results published by Nelson et al..$^{5}$ are used here for analysis. An interference fit of a thick-wall cylinder and a thin-wall shell was made to generate a residual stress of $\sigma_{\max }=186 \sim 200 \mathrm{Mpa}$ along the $x$-axis (the tangential direction of the shell). The test parameters and elastic constants included a wavelength $\lambda$ of $0.5145 \mu \mathrm{m}$, a sensitivity vector $\vec{K}$ of $0.917 \vec{i}+1.40 \vec{k}$, a hole diameter $D_{0}$ of $1.68 \mathrm{~mm}$, a hole depth $H$ of 1.2, a Poisson's ratio $\nu$ of 0.33 and an elastic modulus $E$ of $71 \mathrm{Gpa}$.

The interference fringe pattern obtained for this example was published in Fig. 11 of Ref. 5. Taking $D_{i 1}=2.0$, $D_{i 2}=2.83(i=1,2,3)$, the relative displacements obtained from Tables 1-5 were $\Delta U_{m i}=0.1855, \Delta U_{s i}=0.4150$, $\Delta V_{s i}=0.1604, \Delta W_{m i}=0.0796$ and $\Delta W_{s i}=0.1660 \mathrm{~mm}$. In addition, $\alpha_{1}, \alpha_{2}$ and $\alpha_{3}$ were selected to be 0,63 , and -62 $\mathrm{deg}$, respectively. The fringe order $\left[\Delta N_{i}\left(p_{i 1}, p_{i 2}\right)\right]$, counted directly from the figure, was $[2.5,0.0,0.0]^{t}$. By putting the above data into eqs (5), (2) and (1), we have $\sigma_{\max }=190$ $\mathrm{Mpa}, \sigma_{\min }=-38 \mathrm{Mpa}, \theta=0 \mathrm{deg}$.

In this example, $D_{11}$ was also changed to 2.24 , the relative displacement constants $\Delta U_{m 1}, \Delta U_{s 1}, \Delta V_{s 1}, \Delta W_{m 1}$ and $\Delta W_{s 1}$ were then changed to $0.1176,0.2656,0.0869$, 0.0533 and $0.1023 \mathrm{~mm}$, respectively. The fringe order $\left[\Delta N_{i}\left(p_{i 1}, p_{i 2}\right)\right]$ counted from the published figure was [1.5, $0.0,0.0]^{T}$. Equations (5), (2) and (1) were used to calculate the residual stresses to yield $\sigma_{\max }=180 \mathrm{Mpa}, \sigma_{\min }=-36$ $\mathrm{Mpa}, \theta=0$ deg.

\section{Suggestions}

It is evident from the above two examples that the measured residual stresses obtained from the combined use of the more general form and the holographic blind-hole method are in good agreement with the theoretical values. A question that deserves more attention is the determination of the error in the measured stresses $\left[\delta c_{j}\right]$ when noise prevents the fringe orders from being measured precisely. Even though it is difficult to provide a quantitative answer to this question, the upper bound evaluations are useful for examining measurement errors.

To further examine the measurement errors, eq (5) was replaced by a system of simultaneous linear equations shown below:

$$
\left[a_{i j}\right]\left[c_{j}\right]=\left[N_{i}\right] \lambda,
$$

where $i, j=1,2,3 ;\left[a_{i j}\right]$ and $\left[N_{i}\right]$ represent the square and the column matrices, respectively. Equation (9) can be rewritten as

$$
\left[c_{j}\right]=\left[a_{i j}\right]^{-1}\left[N_{i}\right] \lambda,
$$

where $\left[a_{i j}\right]^{-1}$ denotes the inverse matrix of $\left[a_{i j}\right]$. The measurement errors $\left[\delta c_{j}\right]$, which are induced by the errors of reading the fringe orders $\left[\delta N_{i}\right]$, can be expressed as

$$
\left[\delta c_{j}\right]=\left[a_{i j}\right]^{-1}\left[\delta N_{i}\right] \lambda
$$

for $i, j=1,2,3$. Next, we introduce the norm of a vector $\left[\delta N_{i}\right]$ as

$$
\left\|\left[\delta N_{i}\right]\right\| \equiv\left(\left(\delta N_{1}\right)^{2}+\left(\delta N_{2}\right)^{2}+\left(\delta N_{3}\right)^{2}\right)^{1 / 2},
$$

and the norm of a matrix $\left[a_{i j}\right]$ as

$$
\left\|\left[a_{i j}\right]\right\| \equiv\left(\max \text { eigenvalue of }\left[a_{i j}\right]^{T}\left[a_{i j}\right]\right)^{1 / 2},
$$

where $\left[a_{i j}\right]^{T}$ represents the transposed matrix of $\left[a_{i j}\right]$. Simple manipulations ${ }^{9}$ yield

$$
\left\|\left[a_{i j}\right]^{-1}\left[\delta N_{i}\right]\right\| \leq\left\|\left[a_{i j}\right]^{-1}\right\|\left\|\left[\delta N_{i}\right]\right\| .
$$

Substituting eq (14) into eq (11) leads to

$$
\left\|\left[\delta c_{j}\right]\right\| \leq\left\|\left[a_{i j}\right]^{-1}\right\|\left\|\left[\delta N_{i}\right]\right\| \lambda .
$$

Equation (15) shows the magnitude of measurement error resulting from the errors in reading the fringe orders. This equation also indicates that the smaller the norm of $\left[a_{i j}\right]^{-1}$ the better the measurement accuracy.

A simple numerical program was used to evaluate the norms of $\left[a_{i j}\right]^{-1}$ in eq (5) for variables $\alpha_{1}=m \cdot 15$ deg, $\alpha_{2}=\alpha_{1}+n \cdot 5 \mathrm{deg}, \alpha_{3}=\alpha_{1}-n \cdot 5 \mathrm{deg}$ and $\vec{K}=\cos (l \cdot 15 \mathrm{deg}) \vec{i}+(1+\sin (l \cdot 15 \mathrm{deg})) \vec{k}$, where $m$, $n$, and $l$ are integers, and $m=-5, \ldots,-5, n=1, \ldots, 18$ and $l=0, \ldots, 6$. During the calculations, the relative displacement constants were also obtained from Tables 1-5 with $D_{i 1}=2.0, D_{i 2}=3.0(i=1,2,3), H=1.0$ and $\nu=0.3$. The minimum norms of $\left[a_{i j}\left(p_{i 1}, p_{i 2}, \alpha_{i}\right)\right]^{-1}$ lie on the conditions listed in Table 8. This table is presented as the suggestions for the applications of the general form.

\section{Discussion}

The sensitivity vector for the more general form presented in this article was determined from the illumination and viewing directions. It is suggested that the viewing direction be set normal to the object surface to prevent the original circular hole boundary from transforming into an elliptical hole boundary. Some other kinds of sensitivity vectors may also be adopted in the applications of this form. For instance, the sensitivity vector of the dual-illumination speckle interferometer, ${ }^{10}$ or ESPI, is independent of the viewing direction.

There is another special form, not discussed here, that might be useful for detecting residual stresses. Choosing $\vec{K}=2 \vec{k}$ (normal illumination and viewing), it is clear that only the out-of-plane displacement field contributes to the fringe patterns. Referring to eq (3), the radial line with maximum out-of-plane displacement is consistent with the direction of maximum principal stress. 


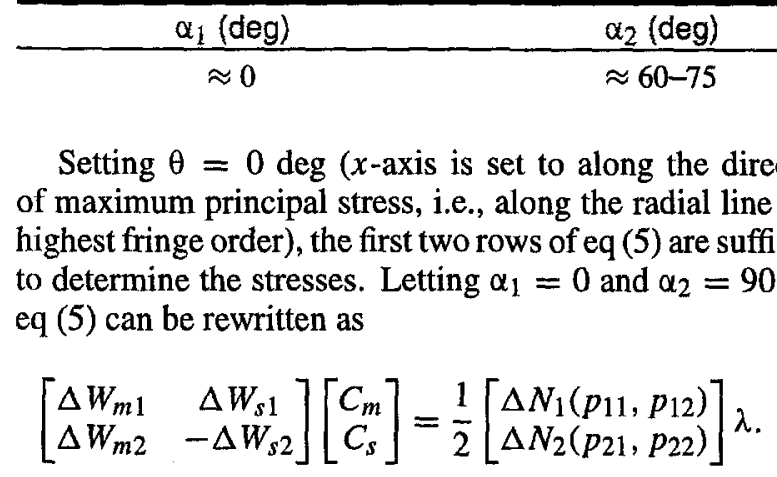

Since there is no need to interpolate the fringe orders while adopting this special form, perhaps it is more appropriate to adopt this form when integrating the large shearing shearography method ${ }^{11}$ with the blind-hole method.

\section{Conclusions}

A more general form for calculating residual stresses detected using the holographic blind-hole method has been presented in this paper. Experiments were performed to prove the usefulness of this form. Suggestions for the application of this form were also established and are shown in Table 8.

\section{Acknowledgment}

The authors greatly appreciate National Science Council of ROC, which supports this research.

$\begin{array}{cc}\alpha_{3} \text { (deg) } & \vec{K} \\ \approx-\alpha_{2} & 0.866 \vec{i}+1.5 \vec{k} \text { to } 0.5 \vec{i}+1.866 \vec{k}\end{array}$

\section{References}

1. Antonov, A.A., "Inspecting the Level of Residual Stresses in Welded Joints by Laser Interferometry," Weld Prod., 30 (9), 29-3I (1983).

2. Bass, J.D., Schmitt, D., and Ahrens, T.J., "Holographic in situ Stress Measurements," Geophys. J. R. Astr. Soc., 85 (1), 13-41 (1986).

3. Nelson, D.V. and McCrickerd, J.T., "Residual-stress Determination Through Combined Use of Holographic Interferometry and Blind-hole Drilling," EXPERIMENTAL MECHANICS, 26 (4), 371-378 (1986).

4. Makino, A. and Nelson, D., "Residual-stress Determination by Singleaxis Holographic Interferometry and Hole Drilling-Part 1: Theory," EXPERIMENTAL MECHANICS, 34 (1), 66-78 (1994).

5. Nelson, D., Fuchs, E., Makino, A., and Williams, D., "Residualstress Determination by Single-axis Holographic Interferometry and Hole Drilling-Part lI: Experiments," EXPERIMENTAL MECHANICS, 34 (1), 79-88 (1994).

6. Lin, S.T., Hsieh, C.T., and Hu, C.P., "Two Holographic Blind-hole Methods for Measuring Residual Stresses," EXPERIMENTAL MECHANICS, 34 (2), 141-147 (1994).

7. Hsieh, C.T., Lin, S.T., Lee, C.K, and Hu, C.P., "Phase-shifting Holographic Blind-hole Method for Measuring Residual Stress with One Axisymmetric Fringe Pattern," Exp. Techniques.

8. Gere, J.M. and Timoshenko, S.P., Mechanics of Materials, 2nd ed., Wadsworth International (1985).

9. Matsumoto, T., Iwata, K., and Nagata, R., "Measuring Accuracy of Three-dimensional Displacements in Holographic Interferometry," Appl. Optics, 12 (5), $961-967$ (1973). 1979).

10. Vest, C.M., Holographic Interferometry, John Wiley, New York

11. Hung, Y.Y., Long, K.W., Wang, J.Q., and Hovanesian, J.D., "Automated Measurement of Residual Stress by Phase-shift Shearography," $A b$ stract Proceedings of the VIII International Congress an Experimental Mechanics and Experimental/Numerical Mechanics in Electronic Packaging, Section II, 122-123 (1996). 